\title{
Acoustic Inversion of the Cold Water Filaments Off the Southwest Coast of Portugal
}

\author{
Paulo Felisberto \\ Sérgio Jesus \\ Institut for Systems and Robotics \\ Universidade do Algarve \\ Campus de Gambelas \\ 8005-139 Faro \\ Portugal \\ Email: \{pfelis,sjesus\}@ualg.pt
}

\author{
Paulo Relvas \\ CIMA \\ Universidade do Algarve \\ Campus de Gambelas \\ 8005-139 Faro \\ Portugal \\ Email: prelvas@ualg.pt
}

\begin{abstract}
Cold water filaments have important implications in the biological and chemical exchanges between the coastal and offshore ocean. The Cape São Vicente area in the Southwest coast of Portugal is a well know region where such phenomenon is observed. In October 2004, the multidisciplinary project ATOMS, involving oceanographers and acousticians, was conducted with the objective to complement the sea surface temperature (SST) satellite observation with a full water column characterization. Due to weather and technical conditions during the project sea trial, only CTD measurements in upper layers of the water column were performed. These at sea collected data together with archival data from the NODC database, allowed to establish a realistic scenario of the $3 D$ temperature distribution in the area, including deeper water layers. Archival data of temperature profiles suggest the occurrence of other important oceanic phenomena such as the subduction of warm Mediterrenean water, that should also influence the acoustic propagation. With the help of forward acoustic modelling the significance and signature of the individual oceanographic phenomena on the acoustic propagation, regarding different sampling strategies of the area by acoustic means is investigated. These investigations allowed to develop strategies to settle the main problem addressed by this work: invert the cold water filament structure by acoustic means in a complex environment where acoustic propagation is affected also by other important oceanic and bathymetric features. Since, the objective of this work, is to evaluate the ability to perform a 3D characterization of vertical structure of the ocean, a minimal transmit-receive acquisition composed of a suspended source from a ship and a drifting vertical array, is assumed. The spatial structure is obtained by a combination of inversions for "mean" sound speed/temperature perturbations obtained for source-array cross-sections covering the area of interest. Matched-field and ray tracing based tomography techniques are used in the inversion for the "mean" perturbations. The planned sampling strategies and necessary acoustic equipment to resolve such oceanic features is discussed having in mind future sea trials.
\end{abstract}

\section{INTRODUCTION}

The occurrence of upwelling giving rise to cold water filaments in Cape São Vicente, Southwest coast of Portugal, is a well known phenomenon, readily observed from satellite sea surface temperature (SST) images. Several oceanographic modelling studies and sea trials were conducted in order to characterize the conditions of the development of such a phenomena, its induced perturbations of the temperature/sound speed in the water column and their spatial extend [1], [2]. It is expected that the strong hydrodynamic activity due to upwelling [3] has implications on the temperature perturbations in the water column and their spatial distribution. In order to study different aspects of the upwelling cold water filaments a group of researchers in various fields (oceanography, biology, chemistry and acoustics) were engaged in the project Acoustic Tomography Acquisition System (ATOMS).

In the framework of the ATOMS project the main goal to be accomplished by acousticians was to develop a simple acquisition system and associated inversion methods to get, in a reasonable time, a "picture" of the filament. The quality of the "picture" found by acoustic means has to be collated with classical oceanographic surveys using CTD. Although, the acquisition system was developed and tested at sea [4], and the applicability of matched field inversion methods to tracking filaments were studied [5], due to logistic problems and weather conditions it was not possible during the project extend to sample the interest area with acoustic means.

In late October 2004, a season when the occurrence of upwelling is unlikely, satellite images of SST showed the presence of a relatively well formed cold water filament. A set of 42 CTD stations with nutrients and chlorophyll water sampling was carried out from the Portuguese navy research vessel NRP D. Carlos I [3], [6]. These direct measurements fairly well characterize the temperature and salinity fields in the upper $400 \mathrm{~m}$ of the water column. Combining these at sea collected data together with archival data from the NODC database, and bathymetric data, allowed to find a more realistic scenario of the 3D temperature distribution in the Cape São Vicente area than this considered in previous work [5]. It includes accounting for the influence in acoustic propagation of other important oceanic phenomena occurring in deeper water layers, such as the subduction of warm Mediterrenean water suggested by archival data and the irregular bathymetry of the area.

This paper is organized as follows. Section II is devoted to find a realistic oceanographic scenario of a cold water filament in the Cape Sao Vicent area. In section III studies, 


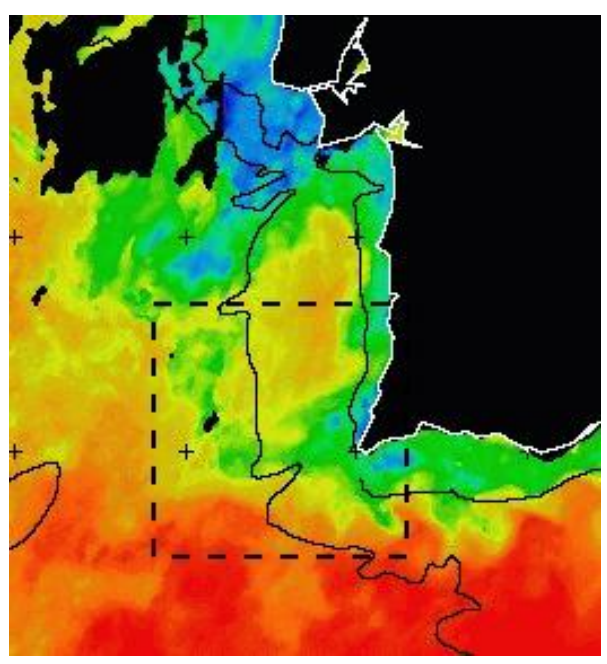

Fig. 1. SST image of SW Portugal acquired 23th October 2004. A cold water filament is observed in the dashed square area. Courtesy from NERC Remote Sensing Data Analysis Service - Plymouth Marine Laboratory, UK.

by simulations, the implications of such a oceanographic process in acoustic propagation. Section IV addresses the problem of inverting a cold water filament by acoustic means with a simple system. Finally, sections V presents the main conclusions of the work.

\section{A FILAMENT SCENARIO}

In order to setup a realistic scenario of a cold water filament in the Cape São Vicent conditions, the following data sets were used:

- an SST image was used to decide where to conduct CTD measurements during the sea trial;

- a set of CTD casts that sample the upper $400 \mathrm{~m}$ of the water column;

- sound speed profiles available in the NODC World Ocean database'98 (WOD'98) for the area, used to setup a baseline environment bellow 400m;

- bathymetric data from the GEBCO ${ }^{1}$ database, and bottom type information from charts.

Next the different data sets used to setup the synthetic scenario are described and the resulting scenario is presented.

\section{A. SST and CTD observations}

Figure 1 shows the SST image acquired on 23th October 2004 where a cold water filament can be observed in an area delimited by the dashed square.

Between 23th and 25th October 2004 a sequence of 42 CTD casts were performed in the area of interest. The sites of the different casts are represented in fig. 2. The acquired sound speed profiles and the corresponding mean profile are shown in fig. 3a), where one can observe that the different profiles have similar shape but suffer from important vertical displacements from the mean profile, what can be associated with a signature of a cold water filament.

\footnotetext{
${ }^{1}$ http://www.ngdc.noaa.gov/mgg/gebco/
}

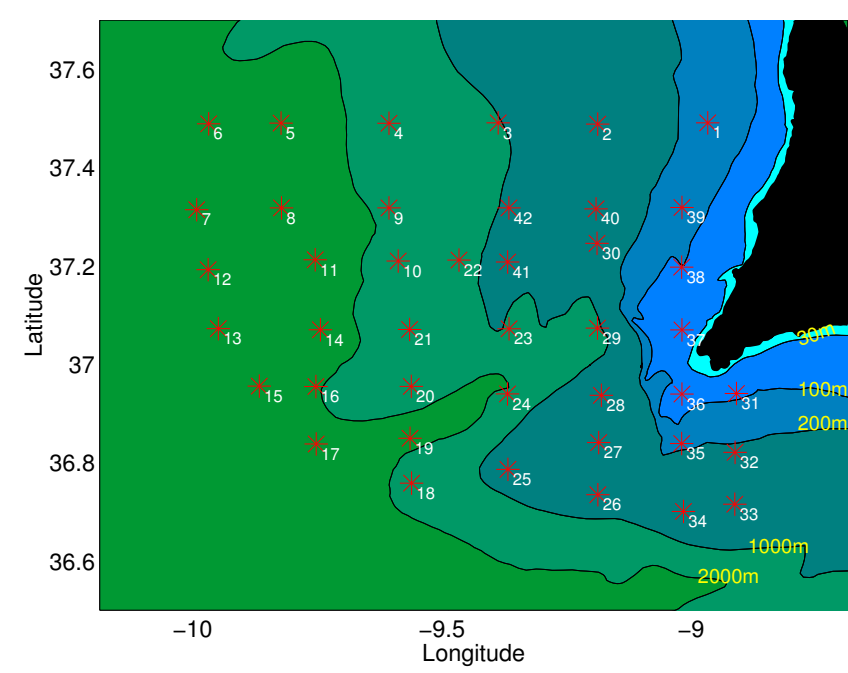

Fig. 2. CTD stations: red $*$ shows the location, the label represents the sequence number of acquisition. The yellow labels are the depth of the bathymetric lines represented in the figure. a)

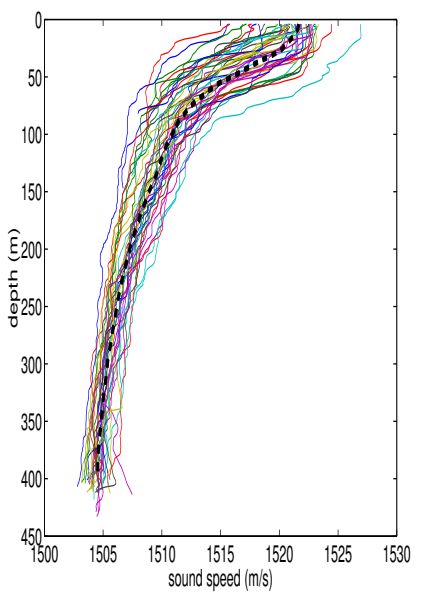

b)

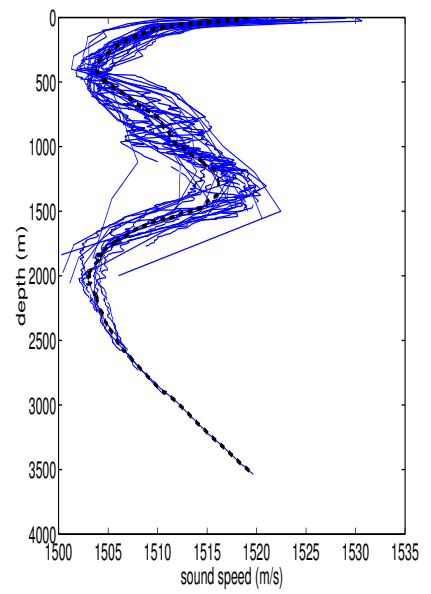

Fig. 3. Sound speed profiles: a) obtained from CTD between 23th and 25th October 2004; b) from WOD'98, acquired during the summer period (from May to October). The black dashed lines represent the mean profiles of the respective data set.

\section{B. Archival data}

Since CTD data covers only the upper $400 \mathrm{~m}$ of the water column, archival data from the WOD' 98 is used to characterize the sound speed profiles bellow this depth until the sea bottom, which at some places in the area reaches $2500 \mathrm{~m}$. From the few samples available in the database for this area, it was considered only samples acquired in the summer period (herein from May to October) where the occurrence of cold water filaments is more likely. Since one needs to characterize the deeper layers, only samples that cover at least the upper $1500 \mathrm{~m}$ were considered. This data is shown is fig. 3b) with the mean profile represented by the black dashed curve. The data was acquired by different instruments (CTD, XBT, among others) until early nineties. Some outliers are observed, but a trend is well defined, which is enough for the proposes of 
TABLE I

CHARACTERISTIC VALUES USED TO SOUND SPEED INTERPOLATION IN DEEPER LAYERS

\begin{tabular}{|c|c|c|}
\hline depth (m) & sound speed (m/s) & comment \\
\hline \hline 1310 & 1516.3 & local maximum \\
\hline 2030 & 1502.8 & local minimum \\
\hline 2488 & 1507.9 & deepest point \\
\hline
\end{tabular}

this work. One can remark that the archival data are inline with the CTD observations discussed in previous subsection. It is also straightforward to see that these profiles show two well localized local minima, near $500 \mathrm{~m}$ and $2000 \mathrm{~m}$ and a local maximum around $1300 \mathrm{~m}$ which may result from other relevant oceanic processes, such the Mediterranean outflow in deeper layers [2]. From the point of view of this work it is important to remark that the shape of sound speed profiles suggest that the sound speed in layers bellow the $400 \mathrm{~m}$, specially until the local maximum around $1300 \mathrm{~m}$, would definitively influence the sound speed propagation whatever will be the position of the sound source and the receivers in the upper layers. This will be shown by simulations in section 3 .

\section{The synthetic scenario}

In order to find a synthetic scenario of the sound speed distribution in the cold water filament area, the CTD observations were objective analyzed (interpolated) using the package EasyKrigV3.0. Figure 4a) presents the spatial distribution of sound speed at $45 \mathrm{~m}$ depth showing a well defined range dependent sound speed perturbation along the 9.5 meridian, which is seen in detail in b) (only upper $450 \mathrm{~m}$ of the water column). When comparing this figure with the SST image (fig. 1) one can see that the spatial distribution of the sound speed at depth $45 \mathrm{~m}$ has the same shape as the surface temperature distribution, what is in agreement with different observations of cold water filaments [1], [5]. The vertical section of fig. 4b) shows a cold water region (reduced sound speed), surrounded by warmer regions (increasing sound speed). As suggested by [2], the upwelling signature is only in the upper ocean layers. Thus, for the remaining of this work it will be assumed that the deeper layers are characterized anywhere by the mean profile obtained from archival data. The complete scenario is shown in fig. 5, where the sound speed in deeper layers, not covered by CTD measurements, were computed by a linear interpolation using characteristic points from the mean profile of the archive data (see table I). The bottom is considered to be covered by mud as reported in hydrographic maps of the region.

This vertical slice of the sound speed distribution will be used next to study the effects of sound propagation in a cold water filament area and settle guidelines to effectively invert it by simple acoustic means.

\section{Simulations ON ACOUSTIC PROPAGATION}

The main objective of these simulations is to determine in which conditions it is possible to invert the perturbations induced by upwelling using a simple acoustic system. The a)

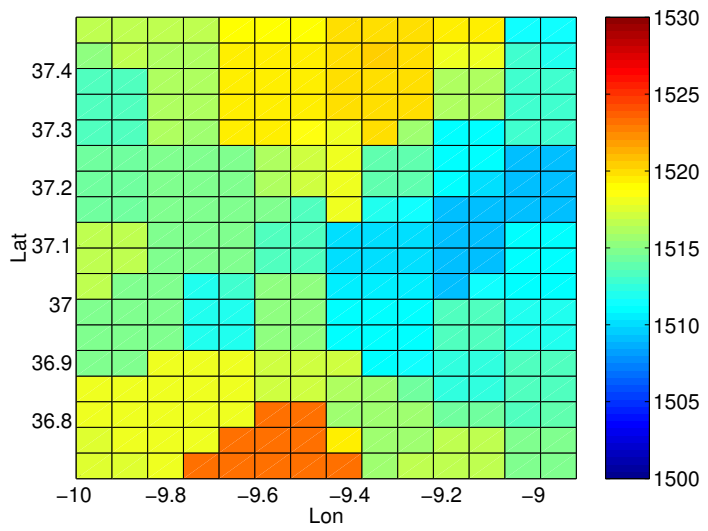

b)

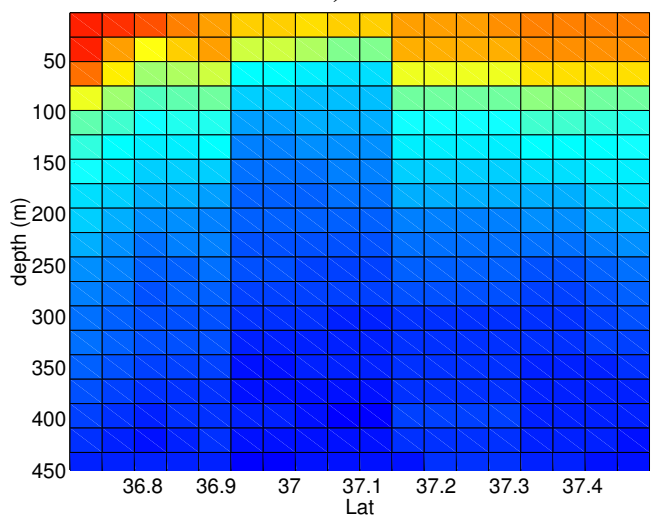

Fig. 4. Spatial distribution of the sound speed in the filament area. a) horizontal slice at $45 \mathrm{~m}$ depth b) vertical slice at longitude -9.5 (upper $450 \mathrm{~m}$ )

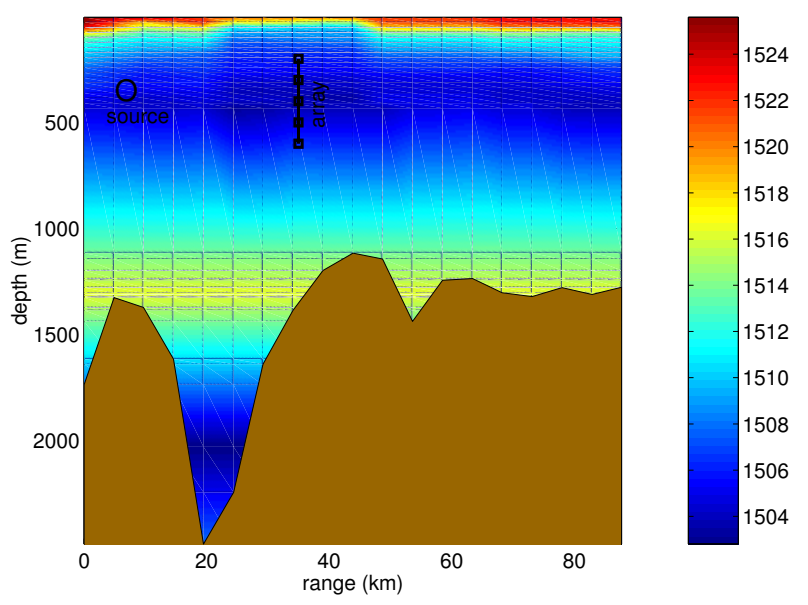

Fig. 5. Synthetic uppwelling scenario for acoustic simulations.

acquisition system considered herein is the Ultra Light Vertical Array (ULVA) reported in ref. [4]. This is an autonomous acquisitions system with local storage (on buoy) and online remote access capabilities, composed of an array of 16 hydrophones and 24 thermistors. It is assumed that the individual hydrophone depths could be adjusted to fit a convenient sampling strategy. A tomographic sound source like that used in previous work [7], [8], emitting LFM chirps in the band 
a)

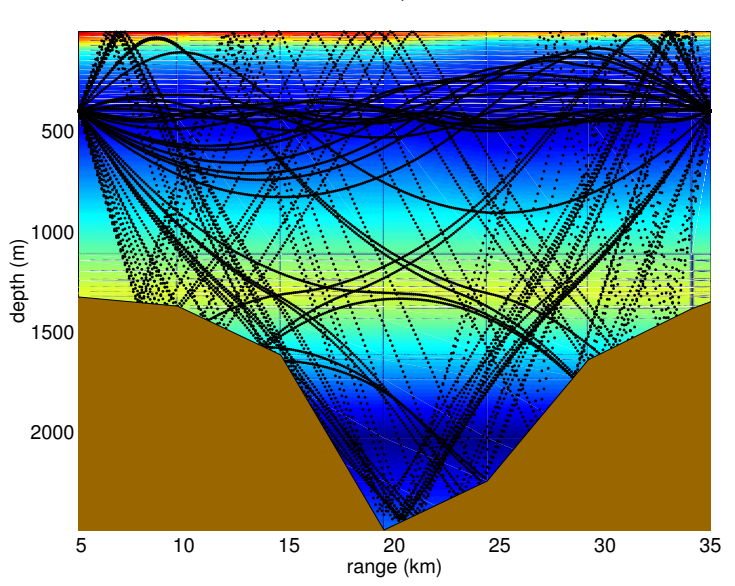

b)

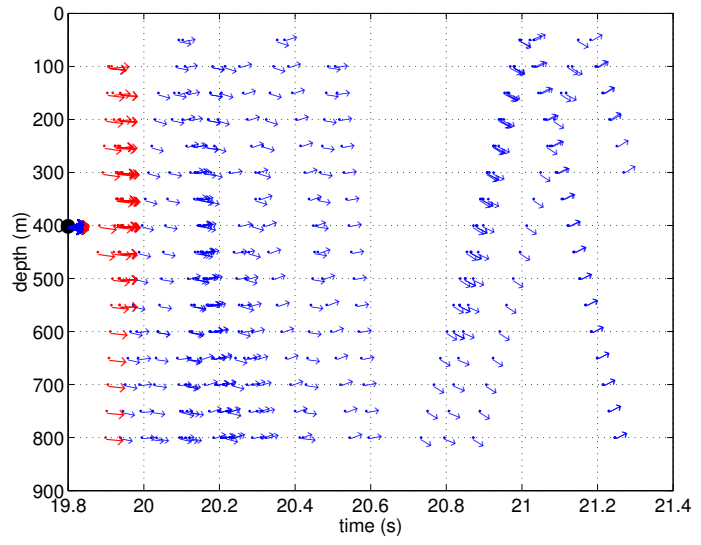

c)

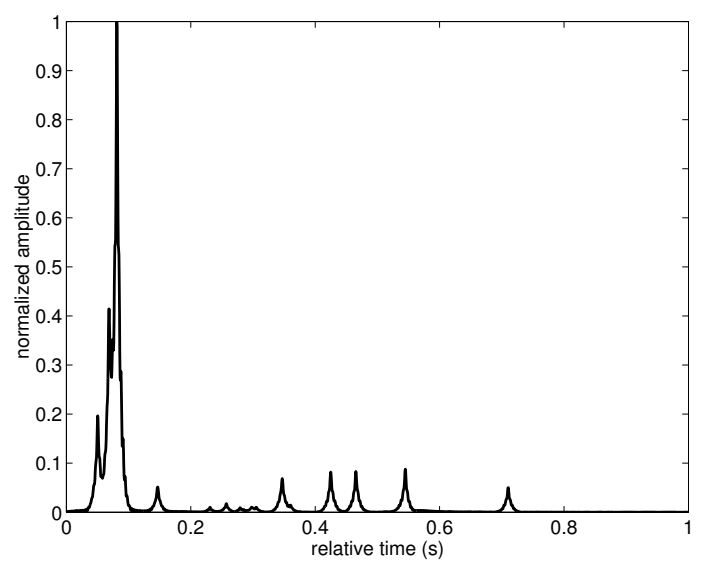

Fig. 6. Sound propagation in a cold water filament using a ray tracing model [9]: a)ray paths between a source and a hydrophone both at depth $400 \mathrm{~m}$; b)arrivals observed at different depths (ranging from 50 to $800 \mathrm{~m}$ with a step of $50 \mathrm{~m}$ ), each arrow represents an arrival and its direction, blue arrows are linked to rays reflected from bottom, red arrows are linked with water refracted rays only; c) arrival pattern for a hydrophone at depth 400m

\section{$300-1000 \mathrm{~Hz}$ is considered.}

The deployment geometry is guided by the satellite SST image so, the array is deployed in the filament (cold water) and the source will be towed by a ship in the surrounding area (warmer water). Thus, one can expect to determine the spatial structure of the filament, by using a set of vertical slice inversions that cover the area of interest as suggested in [8]. Taking into account the vertical slice of the synthetic scenario presented in fig. 5 , where the cold water filament is present in the range $20-40 \mathrm{~km}$, the array is considered to be localized at range $35 \mathrm{~km}$ and the source at range $5 \mathrm{~km}$. The major expected signature of cold water filament is a displacement of the thermocline in the upper layers, the sound source should by placed in these layers, it means in the present case in the upper $400-600 \mathrm{~m}$ of the water column. Figure 6 shows the results of a simulation on the sound propagation in the considered scenario using the ray tracing model RAY [9], being the source and the receiver at $400 \mathrm{~m}$ depth, in a) eigenrays, in b) arrival fronts for the depth interval 50 to $800 \mathrm{~m}$, in c) the arrival pattern. The source and the hydrophone are placed near the sound speed minimum in the upper layer, so it can be seen that the trajectories of the eigenrays with small shooting angles suffer from refraction in layers bellow and above, but close to the source-hydrophone depth. The shape of their trajectories are remarkably influenced by the range dependence of the sound speed. Other eigenrays, although suffering also only refractions, show wider trajectories, which shape are not so much affected by the range dependence (only the travel time is perturbed). It means that the first part of the whole group of rays travels only in filament significant layers, will be tremendously influenced by small changes in sound speed or hydrophone-source geometry. The rays that carry on information about the range dependence of sound speed in the uppwelling significant layers, corresponds to the earlier packet in the arrival pattern. It is straightforward to observe that only a few arrivals within this packet can be separated, moreover their number and strength can vary significantly. The eigenrays rays with larger shooting angles, are in general reflected at the surface and bottom, being influenced also by the irregular bathymetry, bottom type and the sound speed at deeper layers. These rays, corresponding to latter arrivals in the arrival pattern, are strongly attenuated. One should remark that these arrivals do not overlap with those linked with rays refracted in upper layers, thus it allows to isolate the influence of uppwelling and filter out other influences in the received acoustic signal.

Taking into account the reciprocity principle, the shape of expected sound speed profiles specially in the filament significant layers and the arrival diagram presented in fig. $6 \mathrm{~b})$ one can present a guideline to setup the depth of the sound source and the receiver. The sound source and the receiver should be placed in a region where it is likely to occurs the upper local minimum of the different sound speed profiles characteristic of the filament and surrounding area, say between $200-500 \mathrm{~m}$, taking into account the observations and the archival data described in subsections II-A and II-B. If the sound source or hydrophones are placed deeper, it is likely that all rays are reflected in the bottom and the surface and the influence of the uppwelling could not be isolated, as it can be seen for the 50m depth in fig. 6b). Also, no advantage should arise when sound source nor hydrophone is located at a depth 
greater than $500 \mathrm{~m}$, once such a configuration could suffer either from the previously discussed problem, moreover the refracted and reflected arrivals could overlap, as it is suggested by fig. 6b).

\section{FILAMENT INVERSION}

The method suggested to find the spatial distribution of the sound speed (temperature) in a upwelling area is based on the two step procedure proposed by Felisberto et al. [8]. Using this method, the 3D spatial structure of the sound speed distribution is obtained by combining several $2 \mathrm{D}$ sound speed inversions (between an array and a moving source). Moreover, in-situ sound speed measurements, normally acquired at the source and hydrophone array site in order to initialize the acoustic processing could by integrated in the sequel. The method can by readily implemented in an incremental way allowing to obtain an "image" update, that can be used to promptly adjust the sampling strategy.

Next, it will briefly described the method to combine the $2 \mathrm{D}$ sound speed inversions to get a $3 \mathrm{D}$ picture. Also, it will be addressed the inversion for the "mean" sound speed of the 2D vertical slice discussed on acoustic simulations (fig. 6). This vertical slice is highly range dependent with remarkable sound speed profile displacements between adjacent warmer and colder waters. Thus, it is expected that the inversion for a "mean" sound speed profile of this vertical slice represents the more challenging situations that could occur for any considered set of 2D slices that conveniently cover the filament area. Once obtained the 2D inversions, the method to find the $3 \mathrm{D}$ picture of the spatial perturbation of the sound speed is straightforward. Its applicability for an alike perturbation was discussed in [8].

\section{A. Method overview}

In the framework of the two step inversion method the first stage is devoted to find the "mean" sound speed perturbations for different vertical slices of the area of interest, whereas in the second stage the spatial structure of the sound speed perturbations is estimated based on the information obtained in the first step, i.e., a vector of range independent inversions. The first stage is highly problem dependent and for the present case will be detailed in a next subsection, where a perturbative approach based on classical travel time tomography (TTT) [10] and an approach based on arrival pattern matching (MFT) [7] will be addressed. Herein, it is briefly discussed the formulation of the second stage in the light of the current conditions. It is assumed that the first empirical orthogonal function (EOF) obtained from in-situ measurements in the upper layers (says until $1000 \mathrm{~m}$ depth) is sufficient to characterize the sound speed perturbation induced by the upwelling. This a reasonable constraint since the first EOF obtained from data discussed in II-A accounts for more then $66 \%$ of the sound speed perturbation. Also, in the present scenario where it is assumed the deployment of the array in the cold water and the source in the warmer water (guided by the SST image), in-situ measurements of the sound speed are available at both sites, one can compute a significant first EOF from the first cross section considered, that can be updated as new in-situ measurements are available. Thus, it is assumed that the "mean" sound speed perturbation estimated in the first stage for the $j$-th slice $\tilde{\mathbf{c}}_{j}(z)$ is expressed by first EOF coefficient $\tilde{\alpha}_{j}$,

$$
\tilde{\mathbf{c}}_{j}(z)=\tilde{\alpha}_{j} \phi(z)
$$

where $\phi(z)$ is the first EOF. In the second stage, one assumes that the area of interest is discretized into $K$ horizontal cells, with the objective to find the EOF coefficient $\alpha_{k}$ of each cell, $\alpha=\left[\alpha_{1}, \alpha_{2}, \ldots \alpha_{K}\right]$, from the estimates of $J$ slices $\tilde{\alpha}=$ $\left[\tilde{\alpha}_{1}, \tilde{\alpha}_{2}, \ldots, \tilde{\alpha}_{J}\right]^{T}$ found in the first stage. If a linear model is assumed, the second stage can be written as

$$
\tilde{\alpha}=\mathbf{L} \alpha+\mathbf{n}_{i n v},
$$

being $\mathbf{L}$ the observation matrix and $\mathbf{n}_{\text {inv }}$ the errors, where an important component is linked to the first stage inversion. The observation matrix $\mathbf{L}$ has dimension $J \times K$. If the noise is ignored then the $j$-th row of $\mathbf{L}$ in (2) can be written as

$$
\tilde{\alpha}_{j}=l_{j, 1} \alpha_{1}+\ldots+l_{j, k} \alpha_{k}+\ldots+l_{j, K} \alpha_{K} .
$$

The weighting coefficients $l_{j, k}$ can be obtained considering that they represent the weight of rays crossing the $k$-th cell regarding a set of eigenrays computed from the $j$-th mean sound speed perturbation found in the first stage [8]. In this framework, the in-situ measurements can be easily integrated. Assuming that $\alpha_{s}$ represents the vector of measured sound speed perturbations, $\alpha_{m}$ is the vector of true sound speed perturbations at the measurement locations and $\mathbf{n}_{\mathbf{s}}$ represents the measurement errors, than one can expand (2) as follows

$$
\left[\begin{array}{c}
\alpha_{s} \\
\tilde{\alpha}
\end{array}\right]=\left[\begin{array}{cc}
\mathbf{I}_{s} & \mathbf{0}_{s} \\
\mathbf{0}_{l} & \mathbf{L}
\end{array}\right]\left[\begin{array}{c}
\alpha_{m} \\
\alpha
\end{array}\right]+\left[\begin{array}{c}
\mathbf{n}_{s} \\
\mathbf{n}_{\text {inv }}
\end{array}\right],
$$

where $\mathbf{I}_{s}$ is an identity matrix and $\mathbf{0}_{s}, \mathbf{0}_{l}$ are null matrices. System (4)can be solved by different methods [8], [10], and depending on the method used an estimate of the "uncertainty" of the result could be obtained. It is also straightforward to see that the second stage can be implemented in an incremental mode, for example the observation matrix could be simple updated by insertion of a new row each time a new first stage estimate is available.

\section{B. Inversion of a filament cross-section}

Once a convenient set of $2 \mathrm{D}$ inversions is available, the method described above to find spatial distribution of the sound speed, can be, in principle, applied to any environment. Thus, these 2D inversions, which are generally problem dependent are a key issue to get a reliable final result. Next, a TTT and a MFT based method to invert vertical slices of a filament is discussed, keeping in mind that one looks for a "mean" value of the sound speed represented by an EOF coefficient. In both cases, it is considered that the positioning of the hydrophones and the sound source are known. Also, in the TTT based method, it is assumed that synchronization between source and hydrophones allows to obtain absolute 


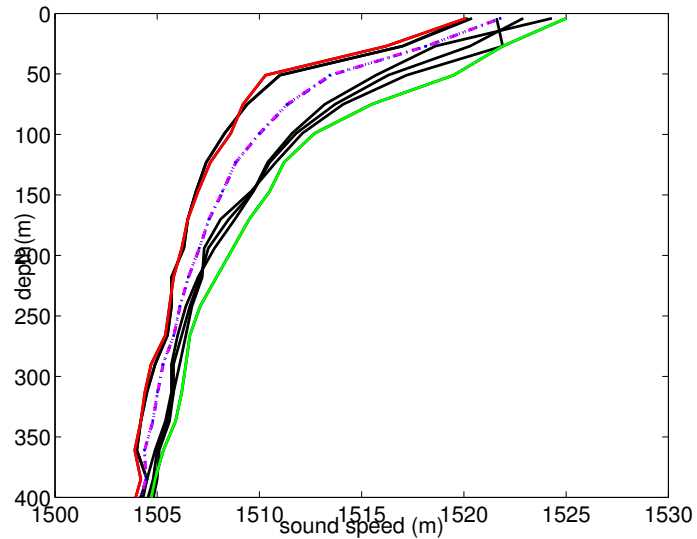

Fig. 7. Inverted sound speed profiles: by TTT blue dashed, by MFT magenta dashed. The other curves represent in-situ sound speed profiles between the source and the array, while the red curve is the profile at the source and the green curve is the profile at the array.

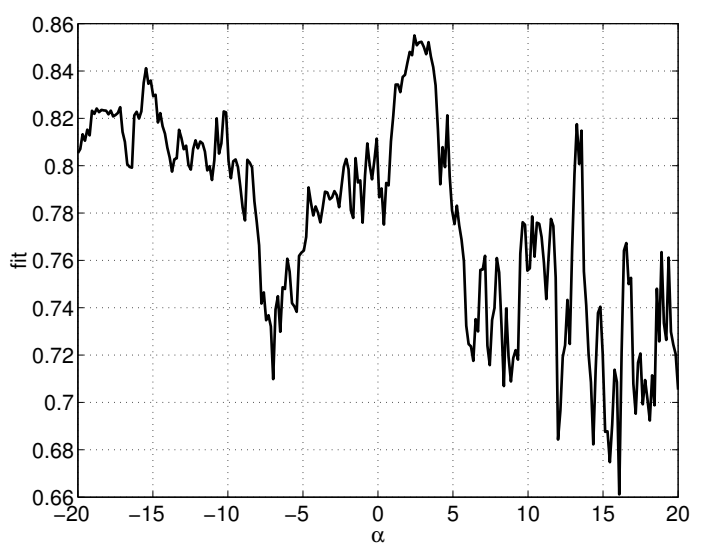

Fig. 8. MFT processor output (fit) dependence on the 1st EOF coefficient

travel times. As an example it is considered the inversion of the vertical slice previously discussed in II-C (see fig. $6)$. It is assumed that the source is deployed at a depth of $350 \mathrm{~m}$ at $5 \mathrm{~km}$ range, and the 16 hydrophone array spawns the water column between 200 and $575 \mathrm{~m}$ at $35 \mathrm{~km}$ range. The sound speed is represented by a mean profile and the $1 \mathrm{st}$ EOF obtained from two sound speed profiles: one acquired at sound source location and other acquired at hydrophone location. One should remark that independently of the method being used, the inversion considers only the first packet of the arrival pattern.

1) TTT based method: Classical TTT methods, based on the relation between rays travel time and sound speed perturbations, are widely used and a detailed description can be found in [10]. The particularity of the method applied herein is that the sound speed is represented by an EOF. One of the most important drawbacks of the TTT method relies in the identification and association of the different rays, as it is suggested in fig. 6c). In this implementation using a very simple algorithm only eight arrivals were identified and could be used in the inversion.
2) MFT based method: The MFT methods are based on the comparison of the received signal with different replicas generated by a model depending on a set of parameters to be estimated. The match between the received signal and the replica for a given parameter set is computed by a so called processor. In the present case the processor computes the geometric average of the maximum of the correlation between the arrival pattern, only first packet, of the received data at each hydrophone and the arrival pattern modelled (replica), being the parameter the coefficient of the first EOF. It is straightforward to see that theoretical maximum achievable by such a processor is the unity, occurring when exists a perfect match between the received data and the replica. One should remark that this method do not need, absolute travel time information, usually technical difficult to implement,

\section{Results}

Figure 7 shows the mean sound speed profiles obtained using the above described methods: blue dashed curve for the OAT method and magenta dashed curve for the MFT method. The other curves in the figure represent the other sound speed profiles between the source and the array, being the red curve the profile at the source and the green curve the profile at the array location. It can bee seen that the inverted profiles are virtually equal. In terms of the EOF coefficient the TTT method estimated a value of 2.55, whereas for the MFT method the estimated value was 2.43 , being the EOF coefficient ranging from -20 to 20 . The estimated values compare with 2.32 , which is the arithmetic mean computed from the considered sound speed profiles between the source and the receiver. It is a pleasant result, since the sound speed profiles is remarkably range dependent with noticeable thermocline displacements, and a visible "mean" equivalent profile were achieved by both methods. Furthermore, the processor used in the MFT method, that is the best suited method for using with simpler acquisition systems, presents good detection and resolution characteristics as suggested by fig. 8 .

\section{CONCLUSION}

In this paper, the problem of inverting a cold water filament in the Cape São Vicente conditions by acoustic means was studied. Based on the analysis of the CTD measurements performed during a sea trial and archival data a representative scenario of the sound speed (temperature) distribution in cold water filament area was developed. The main aspect illustrated by the scenario was: the filament signature occurs in the upper water layers, until aprox. $1000 \mathrm{~m}$, resulting in important vertical displacements of the thermocline between cold and warm waters. The 1 st EOF can be used to effectively parametrize these displacements. In deeper waters the sound speed distribution is affected by other oceanic processes. It is shown that in order to easily filter out the influence of other oceanic processes than the filament in the received signal the sound source and hydrophones should be placed in depths ranging from $200 \mathrm{~m}$ to $600 \mathrm{~m}$. In these conditions it was demonstrated that one can find a "mean" profile for 2D vertical 
slices of the filament region. Moreover, it was shown that a TTT based method and a MFT based method gives comparable results. Since the MFT based method is less demanding for the acquisition system, specially concerning synchronization between emitted and received signal, it is well suited to be applied in light acoustic acquisition systems, as this considered in this work. These 2D inversions can combined using a method proposed by the authors, to find a 3D picture of the filament in an incremental way, giving up to date results that can be used to guide the acoustic sampling strategy.

\section{ACKNOWLEDGMENT}

This work was partially supported by project ATOMS POCTI/MAR/15296/1999 and the FCT Program POSI in the frame of QCA III.

\section{REFERENCES}

[1] P. Relvas, "The physical oceanography of the Cape São Vicente upwelling region observed from sea, land and space," Ph.D. dissertation, University of Wales, Bangor, 1999.

[2] J. Vitorino, "Upwelling off western Iberia (Two case study)," Nice (France), April 2003.
[3] P. Relvas, M. M. A. Cravo, R. Sanchez, and S. Cardeira, "Project ATOMS: characterization of an upwelling filament off Cape São Vicente, SW Iberia," GLOBEC International Newsletter, April 2006.

[4] P. Felisberto, C. Lopes, and S. M. Jesus, "An autonomous system for ocean acoustic tomography," Sea Technology, vol. 45, no. 4, pp. 17-23, April 2004.

[5] V. Corre and S. M. Jesus, "Tracking of cold water upwelling filaments in the ocean using matched field inversion," ACTA ACUSTICA united with ACUSTICA, pp. 604-613, August 2003.

[6] R. F. Sanchez, P. Relvas, A. Peliz, P. Miller, and A. Martinho, "Observation and modelling of an upwelling filament west of cape São Vicente, SW portugal," Vienna (Austria), April 2006.

[7] P. Felisberto, S. M. Jesus, Y. Stephan, and X. Demoulin, "Shallow water tomography with a sparse array during the INTIMATE' 98 sea trial," in Proc. MTS/IEEE Oceans'2003, San Diego (USA), September 2003.

[8] P. Felisberto and S. M. Jesus, "An hybrid acoustic-oceanographic method for estimating the spatial distribution of sound," in Proc. of the 8th European Conf. on Underwater Acoustics (ECUA), Carvoeiro (Portugal), June 2006.

[9] J. Bowlin, J. Spiesberger, T. Duda, and L. Freitag, "Ocaean acoustical Ray-Tracking software RAY," Woods Hole Oceanographic Institution, Tech. Rep., 1992.

[10] W. Munk, P. Worcester, and C. Wunsch, Ocean acoustic tomography, ser. Monographs on mechanics. Cambridge: University Press, 1995. 